\title{
A Curative Perspective on Down Syndrome
}

\author{
Jean A. Rondal
}

University of Liège, Department of Cognitive Sciences, Building 32, Sart Tilman, Liège 4000, Belgium

\begin{abstract}
A curative perspective on Down syndrome is pointing out. Experimental work regarding chromosome correction and corrective action on genes and proteins is yielding positive results. They open the way to advances in dealing with aneuploidies and may end up markedly changing the life of the individuals affected with these conditions at the same time, several molecules are in the research pipeline of cognitive pharmacotherapy. The paper summarizes these advances and set them into perspective for the future of Down syndrome. Research on the effects of the amyloid cascade in the etiology of Alzheimer disease, which is more frequent in aging persons with Down syndrome, is also analyzed. Its potential for improving early diagnosis and paving the way for stabilizing the condition at least in the first stages is also discussed.
\end{abstract}

Keywords: Aneuploidies, Down syndrome, genetic therapy, cognitive pharmacotherapy, Alzheimer disease.

\section{INTRODUCTION}

Human trisomies constitute a significant health problem. Down syndrome (DS) is the most common autosomal trisomy (chromosome 21) occurring in approximately 1 in 700 live births. Increased protein levels due to gene triplication determine a constellation of developmental abnormalities involving the nervous system, the heart, and the intestinal system. Impaired development of the nervous system is the main feature of DS, causing the moderate to severe cognitive handicap typical of the condition.

DS, it should be stressed, is not a disease. It is a genetic condition that may appear in several forms. The most common one ( $95 \%$ of the cases) is standard trisomy 21, and it involves the triplication of chromosome 21 in every body cell. Mosaic trisomy 21 is characterized by the presence of a supplementary chromosome 21 in a part of the cells. In the translocation forms, the long arm of one chromosome 21 or a portion of it is displaced and joined to another chromosome pair $(13,14,15,21$, or 22$)$.

The determinant of standard and mosaic trisomy 21 is meiotic non-disjunction resulting in extra chromosome 21. Translocations involving chromosome 21 may be hereditary and exist in parental genotype although these parents are phenotypically normal) or be de novo occurring in syngamy.

A typical feature of DS is size reduction in frontal and temporal cortices, hippocampus and cerebellum. Maturation abnormalities undermining normal neuronal connectivity are also usually observed.

*Address correspondence to this author at the University of Liège, Department of Cognitive Sciences, Building 32, Sart Tilman, Liège 4000, Belgium; E-mail: jeanarondal@skynet.be
The paper discusses current experimental work in molecular genetics and cognitive pharmacology with a direct bearing on the biology of the condition. These studies open the way to a genuine curative perspective for people affected with Down syndrome

In recent years medical research has made possible to alleviate and in many cases remedy to several of the most current health problems associated with Down syndrome; for example, congenital heart problems, hypothyroidism, auditory and visual deficiencies, atlantoaxial instability, and celiac disorder. Behavioral cognitive rehabilitation has also made important signs of progress in recent times particularly, regarding language and memory. These advances will not be analyzed in the paper as the objective is to concentrate on genetic therapies and applied cognitive pharmacology. The interested reader may want to consider my outgoing book [1] for an extended treatment of the topics discussed in this paper and a few others.

It may seem paradoxical to speak of a possible better future for persons with Down syndrome at a time where many DS associations lament regarding the substantial increases in legal abortions of embryos and fetuses diagnosed with the condition in several Western countries. The advances in the field that will be discussed in this paper may have a positive influence in the middle or longer terms in modifying society's perception and representations of Down syndrome that could begin to be viewed as a curable condition.

\section{GENETIC THERAPIES}

Aneuploidies (i.e., inborn deviations from the normal cohort of 46 chromosomes in humans) have 
traditionally been considered as incurable. However, in recent years, some researchers have attempted to develop therapies applicable to trisomy 21 and other trisomies.

Chromosome correction and reduction of excess gene and protein production contributed by extra Hsa21 (the DS critical zone on the long arm of chromosome 21) have become the object of systematic research work.

\section{Chromosome Correction}

Three experimental techniques have been reported to have met with success in targeting the removal of a human chromosome 21.

Li, Chang, Wang, Hirata, Papayannopoulou, and Russell [2] generated induced pluripotent stem cells (iPSCs) from fibroblasts obtained from adults with DS. Pluripotent stem cells are non-immortalized stem cells that can be turned into a limited series of body cells such as blood, liver, heart or brain cells. Li et al. introduced a TKNEO fusion transgene carried by a modified adenovirus at the locus $21 \mathrm{q} 21.3$ of the gene APP (amyloid-bêta precursor protein) into one copy of chromosome 21. This gene was selected as it is located on the long arm of chromosome 21 and is well expressed in iPSCs.

The fusion of transgene TKNEO with one of the three chromosomes 21 resulted in spontaneous chromosome loss in a large majority of clones, while point mutations, epigenetic silencing, and TKNEO deletions occurred at much lower frequencies in the five experiments undertaken. No damage to other chromosomes in the cells was observed.

Most importantly, the derived, disomic cells proliferated faster than their trisomic counterparts. In a coculture, all disomic cells expanded preferentially, doubling their population on average in about 37 hours against 45 hours for trisomic cells.

$\mathrm{Li}$ et al. warn that genetic manipulation should be undertaken with extreme caution making sure that chromosome deletion does not induce toxicity in the remaining genes and/or a disequilibrium in the rest of the genome. They are confident, however, that their technique could prove useful in clinical applications.

Jiang et al. [3] took advantage of a natural phenomenon to correct trisomy 21. Nature has evolved a particular mechanism to compensate for the difference in dosage of X-linked gene copies between mammalian females and males. In humans, the formula for the sex chromosomes is $X Y$ for males and $X X$ for females. The $Y$ chromosome is much smaller than its $X$ counterpart. It contains only a few dozen genes against about 3000 for the $X$ one.

Natural dosage reduction in females is driven by a ribonucleic acid (RNA), named XIST (for X-inactive specific transcript) that is produced exclusively from the inactive $X$ chromosome. This RNA neither does nor code for proteins. Rather it inactivates the ADN (desoxyribonucleic acid) of the chromosome by methylation turning it into a Barr body.

Jiang et al. reprogrammed fibroblasts obtained from male individuals with DS into iPSCs, and they inserted a transgene XIST at locus 21q22 of the gene DYRK1A in one of the three chromosomes 21. This caused silencing of this chromosome in $88 \%$ of the clones treated. Inactivation of a dozen genes on the inactivated chromosome 21 was further confirmed. No alteration of the other chromosomes was observed. A few sub-clones remained in the 245 colonies of cells treated showing either a chromosome 21 fused with the XIST RNA, two chromosomes 21 in the same state or the three chromosomes 21 fused with the XIST RNA. The authors consider this observation as an indication of the efficiency of their treatment.

As in the experiment by Li et al. [2], the normalized cells exhibited a capacity for proliferation markedly above corresponding trisomic ones.

The authors indicate that the forward-looking implication of their work is to bring Down syndrome into the realm of consideration for gene therapy research. They are confident that the first steps toward this goal have been achieved. They acknowledge, however, that developing a clinical gene therapy is a long process and that much additional work is needed to make it a practical and safe reality.

How does the XIST RNA recognize the particular $X$ chromosome to be inactivated in the ontogenesis of mammalian females? Another gene, antagonist of XIST and named TSIX (an anagram for XIST) has been identified. XIST and TSIX neutralize each other on the $X$ chromosome that remains active, whereas the expression of TSIX is stopped on the $\mathrm{X}$ chromosome to be inactivated [4].

In the experiment by Jiang et al. [3], the fibroblasts were obtained from male persons with DS. It is not 
known what would happen in the same situation using fibroblasts from female persons with DS. Applying the above XIST dosage compensation technique, one could end up either with some cells in the colonies treated exhibiting one chromosome 21 and one $X$ chromosome inactivated, others where one chromosome 21 and the two $X$ chromosomes would be inactivated.

Natural silencing of one chromosome $X$ in females is never complete, and the choice of the genes remaining active is always random. Jiang et al. [3] report, however, that global expressivity of the two active chromosomes 21 in their experiment is between 15 and $20 \%$, which is close to the $22 \%$ usually observed in healthy cells in natural conditions. This suggests that the XIST RNA inserted in the extra chromosome 21 covers key regions of this chromosome, preventing transcription factors from reading the sequence of the nucleic acid.

In contrast to the above complex protocols, Amano, Jeffries, Amano, Ko, $\mathrm{Yu}$, and $\mathrm{Ko}$ [5] undertook to correct aneuploidy by treating cells directly with small molecules.

They first normalized the karyotypes in a culture of mouse embryonic stem engineered to become aneuploid or polyploid, using a biologic made of a mammalian-specific gene, ZSCAN4 (zinc finger - see below - and scan domain) and containing 4 transcription factors expressed in pre-implantation embryos and stem cells., as well as synthetic messenger RNAs (mRNAs) and virus vectors. This suggested that protein ZSCAN4 can determine a marked increase in euploid cells directly.

Amano et al. [5] then tested this biologic on iPSCs generated from fibroblast obtained from four human individuals with DS. Within a few weeks, chromosome examination showed the emergence of up to $24 \%$ and then $40 \%$ of cells with a normal karyotype. The introduction of human ZSCAN4-mRNAs into cells appears to have the power of removing extracopies of chromosome 21 without affecting other chromosomes in the cells.

Similar results were observed with cells obtained from individuals with Edwards syndrome, a genetic condition characterized by triplication of chromosome 18.

Amano et al. [5] speculate that a ZSCAN4 mechanism detects unpaired chromosomes during cell division and separate them from the rest of the replication process. They suggest that an efficient correction of aneuploidy in human fibroblasts is possible using human ZSCANA. Their results justify further work for optimizing doses, delivery, and timing in possible applications of the technique to treat chromosome abnormalities.

\section{Corrective Action on Genes}

Editing genes is possible and has been performed in cases of type B hemophilia and immunity deficiencies. Modified viruses are typically used as vectors.

The "genetic "scissor" CRISPR-Cas9 can perform precise cutting on both the DNA and RNA ribbons. It is now possible for scientists to snip out portions of a chromosome or see what happens when a particular gene is removed. Enzymes that have the power of catalyzing the larger DNA or RNA molecules are employed. Zinc finger nucleases (used by Jiang et al., in work mentioned in the preceding section) are a class of engineered DNA binding proteins exploiting particular properties of the zinc ion. The specific form that it takes at the contact of the DNA molecule motivates its finger name.

Regarding trisomy 21, one could envisage inactivating or removing some of the triplicated genes in Hsa21. Even a modified fraction of this set would determine phenotypic improvement.

A number of genes in Hsa21 show dosage effects: for example, SOD1 (superoxide dismutase-1), DYRK1A (tyrosine Y- phosphorylation kinase), CBS (cystathionine-beta synthase), IFNAR (alpha-interferon receptor), CBR1 and CBR2 (reductase carbonyle), and S100B (glial function in neurons).

Some RNAs have the power to silence any gene in the genome. Wang et al. [6] have documented an association between trisomy 21 and an abnormally low level of protein SNX27 in cells. This protein protects neurons from an excess of the neurotransmitter glutamate. Excess of a particular microRNA (miR-155) linked to the triplication of several genes on chromosome 21 , is correlated with a reduced amount of SNX27. Wang et al. used mice genetically modified to produce fewer proteins SNX27 (named Snx27+/-). These mice showed a learning and memory handicap associated with reduced synaptic recycling of glutamate in spite of globally normal neuroanatomy. 
Synapses operate in conjunction with specific neurotransmitters. They are produced presynaptically and then recuperated postsynaptically. Wang et al. [6] engineered a therapy of replacement at the level of the hippocampus (a brain structure involved in memory) for restoring normal levels of protein SNX27 and postsynaptic glutamate recycling, which yielded better learning and memory in the experimental mice.

Murine models are useful tools in genetic research. Mice Ts65Dn, Ts1Cje, and TgYAC152F7, for example, have been genetically modified to correspond as much as possible to the triplication of Hsa21 in humans. Orthologous genes in mice are distributed on chromosomes 10, 16, and 17. Genetically modified mice develop a phenotype akin to partial trisomy 21 in humans [7].

Fillat and collaborators [8] employed an adenovirus as a vector to carry microRNA within the hippocampus of mice Ts65DN for reducing the expressivity of gene DYRK1A and, separately, a lentivirus to increase the expression of another gene, MECP2 located on the long arm of the X chromosome at the locus Xq28. In both cases, a marked improvement was observed in the learning ability of the experimental mice. This research suggests that genes located on other chromosomes but the 21 may have a role in the phenotype of Down syndrome perhaps in interaction with the Hsa21 genes or some of them.

Acting on genes may not be without risk, however. Our immunological systems have evolved to contrast viral aggression. It is imperative to make sure that reprogrammed cells will not be confounded with infected cells and become targets for destruction. Gene correction in utero will become possible in the future. It should be practiced with extreme caution, however, as major risks exist for mothers and fetuses, given the toxicity of the viruses, possible adverse immunological reactions, and the possibility of tumor generation [9].

\section{Action on Proteins}

Other than modifying genes and manipulating RNAs, it is possible to act on the proteins encoded by these genes.

Much attention has been devoted recently to a molecule named epigallocathechine gallate (EGCG), a polyphenol of green tea with antioxidant properties. It happens to be a potent inhibitor of the enzyme produced by the gene DYRK1A; a gene that plays an important role in neurogenesis and in consolidating memory engrams, but which when overexpressed causes brain toxicity.

Guedl et al. [10] submitted Ts65Dn and Ts1Cje mice carrying an additional copy of gene DYRK1A to a diet rich in EGCG from gestation to adult age. The treated animals developed a normal phenotype as opposed to those in the control group.

Stagni et al. [11] have published an extensive review of the literature on the effects of EGCG in animal and human research. Several studies with genetically modified and transgenic mice for the gene DYRK1A confirm the efficiency of this therapeutic strategy. Restoration of hippocampal development is observed associated with better memory.

However, when treatment is interrupted, the benefits are lost. Early and chronic administration of EGCG appears to be a condition for efficiency. Even higher doses of the product are inefficient when administered later in life.

At the human level, two studies conducted by De la Torre and associates deserve attention. In a first study [12], they reported positive effects on visual recognition in a group of young adults with DS following three months of daily treatment with EGCG (green tea extracts, low in caffeine, $9 \mathrm{mg} / \mathrm{kilo}$ of body weight, administered orally). However, three months after the end of treatment, participants 'performances returned to pre-intervention levels.

In a second study, De la Torre and collaborators [13] tested the effect of a daily EGCG treatment (with a dosage identical to the one in the previous research), lasting for one year, coupled with a behavioral program of cognitive training. The sample of subjects counted 84 young adults with Down syndrome (about as many women as men, aged between 16 and 34 years). They were divided in two groups: one group treated with EGCG and receiving cognitive training; the other group receiving a placebo and the same cognitive training as the first group.

A battery of neuropsychological tests was administered at the end of the study. Participants treated with EGCG and exposed to cognitive training showed statistically significant superiority into two cognitive tests (memory and visual recognition) and adaptive tasks (measuring ability in daily routines). A retest 16 months after the end of the experiment showed only a partial persistence of the effects measured at the end of the intervention. 
The experimental plan of De la Torre et al. in the second experiment does not allow separating the effect of EGCG administered alone, as the molecule and the cognitive training were combined in the first group of subjects. The clinical efficiency of EGCG administered in isolation, and its extent remains to be established.

Several of the studies reviewed suggest the interest in the intervention to proceed early in life. A significant part of the phenotype associated with trisomy 21 is already present before birth. Early intervention, everything else being equal, is more likely to be efficient as it takes place at a time when the organism is still in formation.

Prenatal screening for Down syndrome is possible from the eleventh week of pregnancy through the analysis of foetal DNA fragments present in maternal blood samples. Ninety-nine percent adequacy can be reached in combining blood analysis, the cardiac rhythm of the foetus, and nuchal translucency [14].

These techniques are not diagnostic. A percentage of false positive or false negative may remain. Fully diagnostic techniques are amniocentesis and histomorphologic analysis of chorionic villi. They can be performed between the $15^{\text {th }}$ and the $18^{\text {th }}$ week of pregnancy.

Once rendered safe, prenatal intervention needs to be practiced as early as possible in foetal life. Very early diagnosis of trisomy 21 is a necessity. It could be proposed to parents diagnosed with a translocation involving chromosome 21 . These parents, while being phenotypically normal, have a $100 \%$ chance to give birth to a child with trisomy 21 .

\section{COGNITIVE PHARMACOTHERAPY}

A series of molecules in the pharmacological pipeline appears to have the power to improve cognitive development and functioning in individuals with DS.

Guidi and associates [15] administered fluoxetine, a molecule used in treating depression, to pregnant mice Ts65Dn in continuity from embryonic stage until birth. After delivery, the mice whose mothers had been given fluoxetine exhibited normal neuronal proliferation and dendritic growth in brain areas.

Donepezil is a molecule inhibiting the degradation of the activating neurotransmitter acetylcholine in reducing acetylcholinesterase (which catalyzes acetylcholine). In adults with DS and already in children, atrophy of cholinergic neurons is observed in several brain areas.

Kishnani et al. [16] tested the efficiency of donepezil with young DS adults treated with doses of $5 \mathrm{mg}$ per kilo of weight for 6 weeks and then $10 \mathrm{mg} / \mathrm{kilo}$ for the following 6 weeks. Significant cognitive benefits were measured in the subjects treated as opposed to those having received a placebo. Other works with young DS adults have also reported positive indications following administration of donepezil (Capone [17] for a review).

Kishnani et al. [18] have also tested the efficiency of donepezil with DS children and adolescents (aged between 10 and 17 years). Participants received a dose of $2.5 \mathrm{mg} / \mathrm{kilo}$ donepezil in the first part of the experiment, increased every 14 days until reaching $10 \mathrm{mg} / \mathrm{kilo}$. Significant gains in verbal expression and reasoning were measured in the experimental subjects as opposed to the control group that received only a placebo.

Rivastigmine is a molecule with globally the same properties as donepezil. Its efficiency was tested by Heller and associates in two studies with DS children and adolescents aged between 10 and 17 years [19, 20]. Doses at the beginning of the intervention were $1.5 \mathrm{mg} / \mathrm{kilo}$ increased to $3 \mathrm{mg} / \mathrm{kilo}$ and $4.5 / \mathrm{kilo}$ in the following weeks. After 16 weeks, results showed statistically significant gains in attention, language, memory and adaptive behaviors. Five participants continued the treatment for another 38 months without additional benefit.

Glutamate is the major excitatory neurotransmitter in the central nervous system. It also plays a role in reinforcing and stabilizing neural connections and dendritic growth. Animal experimentation and clinical trials in humans are still lacking in this domain.

Also in the pipeline of cognitive pharmacology, is the GABA systems (for gamma-aminobutyric acid), which is the major brain inhibitory neurotransmitter. Piracetam has motivated much interest in recent years. This molecule has the power to increase brain oxygenation and improve cholinergic, glutamate and GABA neurotransmission. However, clinical trials carried with DS children have not yielded positive indication so far [21].

An antibiotic used to treat acne, minocycline, appears to have a therapeutic property regarding a category of brain cells, the astrocytes (glial cells 
supplying nutrients to the neurons and detoxifying the extracellular milieu in neutralizing excess glutamate). Chen et al. [22] observed that the astrocytes induced in vitro from stem cells obtained from persons with DS do not favor in vivo neurogenesis when transplanted in iPSCs in mice brains. Minocycline corrects this deficiency by modulating the expression of gene S100B, located on chromosome 21 at locus $21 \mathrm{q} 22.3$. This gene regulates various cellular processes, including the glial function.

It may also be of interest to provide the persons with DS with carefully controlled metabolic complements such as lithium, vitamin E, coenzyme CoQ10, or folinic acid [23].

Cognitive pharmacotherapy as applied to DS is still in infancy. But it has the potential to boost neurotransmission.

A more ambitious strategy would be to build "a better brain" early in development. Capone [17] notes: "Biologically assisted neuromaturation could represent the Holy Grail of brain-based intervention for children with trisomy 21. If only we knew how to do this, we could debate if in fact we should, under what circumstances, and the reasons why or why not. Cellbased therapies engender the possibility of directing neurodevelopment along a more sustained promaturational trajectory intent on generating network complexity, enhanced performance, and long-term stability" (p.109). Capone adds that we are not close to testing such ideas in humans. True enough, but the perspectives in genetic therapy are genuine steps in this direction.

\section{ALZHEIMER DISEASE}

A majority of persons with DS show accelerated ageing affecting particularly the immune and central nervous systems. Whether earlier ageing is always predictive of Alzheimer disease (AD) is still a matter of controversy. It is known that standard trisomy 21 is associated with an increased probability to develop AD. This suggests a role for the genes in Hsa21 in the etiology of this degenerative pathology.

Alzheimer disease affects approximately $20 \%$ of DS persons beyond 40 years of age, $40 \%$ beyond 50 years, and close to $80 \%$ beyond 60 years of age. In some cases, the degenerative process is relatively slow going and may cover a dozen years. In other cases, involution is more rapid.
The point of departure of Alzheimer's pathology in Down syndrome is found in a so-called amyloid cascade induced by the overexpression of the gene APP located on the upper part of the long arm of chromosome 21. DS persons with a partial translocation not involving the upper part of chromosome 21 do not present a particular susceptibility to $A D$ while showing most of the $D S$ phenotype. Those with a partial translocation involving the upper part of this chromosome often present an $A D$ pathology.

As soon as the first years of life, considerable quantities of non-toxic amyloid-alpha proteins are produced in the brain of individuals with DS. A phenomenon not yet identified operates switching this production to toxic peptides amyloid-beta 40 and 42 that cannot be dissolved in brain fluids and determine the formation of the amyloid plates.

These plates have an affinity for the junction points between neurons, and they disturb neurotransmission. The aggregates end up circumventing the neuronal bodies. This causes the accumulation within neurons of a protein called TAU responsible for the neuro fibrillation of the cell's cytoplasm, which annihilates their functional ability. The amyloid plates gradually invade all cortical layers, mid-brain structures, the brain trunk, and the cerebellum.

But not all DS persons develop AD. Compensatory mechanisms operate of which almost nothing is known. Other genes on other chromosomes also, but the $21 \mathrm{st}$ chromosome influence the determinism and the course of $A D$ also for non-DS ageing persons. A gene encoding apolipoprotein $E$ (associated with cholesterol metabolism), exists in three allelic variants. APOE E3 is the more frequent in the population, followed by APOE E4 and APOE E2. Higher dosages in APOE 3 and APOE 4 are correlated with an increased and earlier risk for $A D$. It is associated with a higher concentration of protein amyloid-beta in the brain. Variant APOE E2 instead seems to have a protective action [24].

Allelic variants of gene SORT1 (sortilin receptor located on chromosome 1) have been identified as additional risk factors for a later onset of $A D$ [25]. SORT1 plays a role in the elimination of excess amyloid-beta proteins in the brain. When it is underexpressed, one observes an increase in brain amyloid deposits. Vascular cholesterol may also be involved in an interaction with genotype APOE [26]. 
What are the therapeutic perspectives for $A D$ at the present times?

The first molecule proposed for contrasting the early manifestations of $A D$ was tacrine, an inhibitor of enzyme acetylcholine esterase. It was retired from the pharmacological market because of its hepatic toxicity.

Other inhibitors of acetylcholine-esterase have been developed meanwhile with no particular toxicity, for example, donepezil, rivastigmine, and galantamine.

Acetylcholine-esterase reduces acetylcholine to its basic constituents allowing the cholinergic neurons to return to a state of rest following activation.

A moderate cholinergic deficit exists in Down syndrome, as indicated. It is magnified in AD. In mice Ts65Dn, overexpression of the molecule amyloid-beta has been shown to be associated with a degeneration of cholinergic and noradrenergic neurons, particularly in the brain hippocampal region [27]. On this basis, one has proposed to reduce the natural elimination of neurotransmitter acetylcholine with donepezil, rivastigmine, or galantamine. But so far, the attempts to improve cognitive functioning in $D S$ patients with $A D$ have met only with limited success [28].

Memantine is a molecule that controls the activity of the neurotransmitter glutamate and acts on the NMDA (N-methyl-D-aspartate) receptors for regulating the quantity of ions calcium entering neurons in the propagation of the nerve influx. Excess glutamate increases the activity of the NMDA receptors causing an excess of calcium entering in the cells. This alters neural transmission. Clinical trials with memantine so far have also met with limited success in improving cognitive functioning in DS patients with AD [29].

Lithium has been tried by Matsunaga and associates [30] in an attempt to limit cognitive deterioration in DS persons at the first stages of AD. They have reported positive results in comparison with a control group. However, AD incipiens in $D S$ is characterized by episodes of depression. Lithium is known as a mood stabilizer. It is not clear whether the molecule acts mostly as an antidepressant or whether it may constitute a genuine antagonist to early cognitive deterioration in $A D$.

A series of antioxidant molecules, for example, nicotinamide, levocarnitine, and lipoic acid, have been tried for contrasting oxidative stress, thought to play a role in the etiology of $A D$, but, it would seem, without encouraging results so far. Another series of drugs is the object of current experimental research; for example, insulin and immunoglobulin [31].

The gene DYRK1A has been listed as a primary suspect in the etiology of AD. Normalizing DYRK1A dosage by breeding mice Ts65Dn with a triplication of this gene with mice trisomic for the same DNA segment but without the gene DYRK1A, has yielded TS65Dn mice with a normal dosage of this gene and a standard concentration of protein APP in the cerebral cortex, hippocampus, and cerebellum [32].

Kawakubo and associates [33] injected harmine, an inhibitor of protein DYRK1A, in fibroblasts obtained from $D S$ persons with $A D$ pathology. They measured an important increase in the concentration of enzyme neprilysin and correlatively a decrease in the concentration of the DYRK1A protein in the fibroblasts.

Accumulation of amyloid plates in the brain intensifies some 5 to 20 years before a significant cognitive decline is observed. Increased concentration of TAU protein and neuro fibrillation of cells cytoplasm can be objectified 1 to 5 years before a diagnosis of $A D$ is warranted. And a noticeable reduction of cerebral volume in prefrontal cortex and hippocampus can be measured 1 to 3 years before AD diagnosis.

It should be possible to validate a list of biological markers of $A D$, which would allow screening for, and early intervention in $A D$, if only to stabilize the condition. Among the promising candidates in this respect are, concentration of protein APP in blood plasma [34] and the expression of MTRNR21, a mitochondrial gene involved in neuron protection and an antagonist of cell apoptosis [35]. Neurological examination with the technique of transcranial magnetic stimulation (a noninvasive form of brain stimulation able to measure the activity of brain circuits) can reveal synaptic dysfunctions possibly linked to an AD incipiens [36].

A genuine curative strategy for $A D$ would be to prevent the accumulation of amyloid plates, proliferation of TAU proteins, and neurofibrillation of neurons cytoplasm. Several molecules and drugs are being tested, which may prove efficient in these respects, such as the inhibitors of the APP protein, vaccines and antibodies, inhibitors of the TAU protein, as well as a number of other biochemical agents thought to be able to boost brain defenses against APP and TAU toxicity. 


\section{CONCLUSION}

Existing developments are taking place in the field of DS with a perspective of significant improvements in life and cognitive functioning for persons with the condition. Genetic therapy is still largely experimental and restricted to in vitro manipulations. It requires an important number of additional works before being applicable in vivo. But the movement is launched, and there is no reason why, if properly encouraged and funded, it would come to a stop.

Cognitive pharmacotherapy is an active field, not the least regarding intellectual disabilities and Down syndrome. Decisive outcomes have been relatively rare so far. However, a number of molecules are in the research pipeline. It is likely that some of them at least will prove efficient in boosting brain development and neurotransmission in children with Down syndrome.

As briefly mentioned in the introduction, medical research is progressing. It has been successful in reducing the impact of several illnesses affecting persons with DS more often than other persons. Behavioral cognitive research, above all regarding language and memory, has refined its strategies and techniques in recent decades, and there is still room for improvement.

Biological research on Alzheimer disease has identified a significant part of the causal chain leading to brain degeneration. $A D$ in $D S$ and non-DS persons seem to proceed in the same way, even if only DS persons present a triplication of Hsa 21. Research on $A D$ in non-DS aging persons will also benefit DS persons and vice versa. AD represents a very active field of biological and pharmacological research and for good reasons. In the United States alone, at present, there are 4.7 million persons diagnosed with Alzheimer disease. An increase of 40 to $50 \%$ is expected in the coming decades associated with the gains in life expectancy.

Our societies should encourage research aiming at correcting aneuploidies and their phenotypic manifestations.

Lionel Penrose, one of the English pioneers in the study of cognitive handicap, used to say that a society ought to be judged on the way it takes care of its less favored citizens [37]. The coming generations will judge us on that.

\section{REFERENCES}

[1] Rondal JA. Le futur de la trisomie 21. Une perspective curative. Brussels: Mardaga 2019.

[2] Li L, Chang K, Wang P, Hirata R, Papayannopoulou T, Russell D. Trisomy correction in Down syndrome induced pluripotent stem cells. Cell Stem Cell 2012; 11: 615-9. https://doi.org/10.1016/j.stem.2012.08.004

[3] Jiang J, Jing Y, Cost G, Chiang JC, Kolpa H, Cotton A, Carone D, Carone B, Shivak D, Guschin D, Pearl E, Rebar E, Byron M, Gregory P, Brown C, Urnov F, Hall L, Lawrence J. Translating dosage compensation for trisomy 21. Nature 2013; 500: 296-300. https://doi.org/10.1038/nature12394

[4] Lee J, Davidow L, Warshawsky D. Tsix, a gene antisense to Xist at the X-inactivation centre. Nat Genet 1999; 21 : 400-4. https://doi.org/10.1038/7734

[5] Amano T, Jeffries E, Amano M, Ko A, Yu H, Ko M. Correction of Down syndrome and Edwards syndrome aneuploidies in human cell cultures. DNA Res 2015; 22: 331-42. https://doi.org/10.1093/dnares/dsv016

[6] Wang $X$, Zhao $Y$, Zhang X, Badie H, Zhou Y, Mu Y, Loo L, Cai L Thompson R, Yang, Chen Y, Johnson P, Wu C, Bu G, Mobley W, Zhang , Gage F, Ranscht B, Zhang Y, Lipton S, Hong W, Xu $\mathrm{H}$. Loss of sorting nexin 27 contributes to excitatory synaptic dysfunction by modulating glutamate receptor recycling in Down's syndrome. Nat Med 2013; 19: 473-80. https://doi.org/10.1038/nm.3117

[7] Rueda N, Flórez J, Martinez-Cue C. Mouse models of Down syndrome as a tool to unravel the causes of mental disabilities. Neural Plasticity 2012 https://doi.org/10.1155/2012/584071

[8] Fillat C, Bofill-De Ros $X$, Santos M, Martin E, Andreu N Villanueva E, D’Amico D, Dierssen M, Altafaj X. Identification de genes clave implicados en el sindrome de Down mediante terapia genetica. Rev Med Int Sindrome Down 2014; 18(2): 21-8. https://doi.org/10.1016/S2171-9748(14)70049-2

[9] Caplan A, Wilson J. The clinical challenges of in utero therapy Nat Genet 2000; 24: 107-8. https://doi.org/10.1038/72747

[10] Guedj F, Sebrie C, Rivals I, Ledru A, Paly E, Bizot J, Smith D, Rubin E, Gillet B, Arbones M, Delabar, JM. Green tea polyphenols rescue brain defects induced by overexpression of DYRK1A. Plos ONE 2009; 4(2): 1-8. https://doi.org/10.1371/journal.pone.0004606

[11] Stagni F, Giacomini A, Emili M, Trazzi S, Guidi S, Sassi M, Ciani E, Rimondini R, Bartesaghi R. Short- and long-term effects of neonatal pharmacotherapy with epigallocatechin-3-gallate on hippocampal development in the Ts65Dn mouse model of Down syndrome. Neuroscience 2016; 333: 277-301.

https://doi.org/10.1016/j.neuroscience.2016.07.031

[12] De la Torre R, de Sola S, Pons M, Duchon A, De Lagran M Farre M, Fito M, Benejan B, Langohr K, Rodriguez J, Pujadas M, Bizot J, Cuenca A, Janel N, Catuara $S$, Covas $M$, Bléhaut $H$, Hérault Y, Delabar JM, Dierssen M. Epigallocatechin-3-gallate, a DYRK1A inhibitor, rescues cognitive deficits in Down syndrome mouse models and in human. Mol Nutr Food Res 2014; 58: 27888

https://doi.org/10.1002/mnfr.201300325

[13] Sparks A, Truble C, Wang E, Song K, Oliphant A. Noninvasive prenatal detection and selective analysis of cell-free DNA obtained from maternal blood: Evaluation for trisomy 21 and trisomy 18. Am J Obst Gynecol 2012; 206: 319.e1- e9.

https://doi.org/10.1016/j.ajog.2012.01.030

[14] Nicolaides K, Syngelaki A, Poon L, Gil M, Wright D. Firsttrimester contingent screening for trisomy 21, 18, and 13 by biomarkers and maternal blood cell-free DNA testing. Fetal Diagn Ther 2014; 35: 185-92. https://doi.org/10.1159/000356066

[15] Guidi S, Stagni F, Bianchi P, Ciani E, Giacomini A, De Franceschi M, Moldrich R, Kurniawan N, Mardon K, Giuliani A, Calza L, Bartesaghi R. Prenatal pharmacotherapy rescues brain development in a Down's syndrome mouse model. Brain 2014; 137: 380-401.

https://doi.org/10.1093/brain/awt340 
[16] Kishnani P, Sommer B, Handen B, Seltzer B, Capone G, Spiridigliozzi G, Heller J, Richardson S, McRae T. The efficacy, safety, and tolerability of donepezil for the treatment of young adults with Down syndrome. Am J Med Genet 2009; 149: 164154.

https://doi.org/10.1002/ajmg.a.32953

[17] Capone G. Pharmacotherapy for children with Down syndrome. In Rondal JA, Perera J, Spiker D, editors. Neurocognitive rehabilitation of Down syndrome. The early years. Cambridge, United Kingdom: Cambridge University Press 2011; pp. 96-116. https://doi.org/10.1017/CBO9780511919299.008

[18] Kishnani P, Heller J, Spiridigliozzi G, Lott I, Escobar L, Richardson S, Zhang R, McRae T. Donepezil for treatment of cognitive dysfunction in children with Down syndrome aged 1017. Am J Med Genet 2010; 152: 3028-35. https://doi.org/10.1002/aimg.a.33730

[19] Heller J, Spiridigliozzi G, Crissman B, Sullivan J, Eells R, Li J, Doraiswamy P, Krishnan R, Kishnani P. Safety and efficacy of rivastigmine in adolescents with Down syndrome: A preliminary 20-week, open-label study. J Child Adolesc Psychopharmacol 2006; 16: 755-65. https://doi.org/10.1089/cap.2006.16.755

[20] Heller J, Spiridigliozzi G, Kishnani P, Crissman B, McKillop J, Yamamoto $\mathrm{H}$. Safety and efficacy of rivastigmine in adolescents with Down syndrome: Long-term follow-up. J Child Adolesc Psychopharmacol 2010; 20: 517-20.

https://doi.org/10.1089/cap.2009.0099

[21] Lobaugh N, Karaskov V, Rombough V, Rovet J, Bryson S, Greenbaum R, Haslam R, Koren G. Piracetam therapy does not enhance cognitive functioning in children with Down syndrome. Arch Pediatr Adolesc Med 2001; 15: 442-8. https://doi.org/10.1001/archpedi.155.4.442

[22] Chen C, Jiang P, Xue H, Peterson S, Tran H, McCann A, Parast M, Li S, Pleasure D, Laurent L, Loring J, Liu Y, Deng W. Role of astroglia in Down's syndrome revealed by patient-derived human-induced pluripotent stem cells. Nat Com 2014. https://doi.org/10.1038/ncomms5430

[23] Patterson D Development of the brain and metabolism. In Rondal JA, Perera J, Spiker D, editors. Neurocognitive rehabilitation of Down syndrome. The early years. Cambridge, United Kingdom: Cambridge University Press 2011; pp. 85-95. https://doi.org/10.1017/CBO9780511919299.007

[24] Firth N. Startin C, Fisher E, Nizetic D, Kong Chian L, Hardy J, Tybulewics A, Karmiloff-Smith A, Strydom A. Aging related cognitive changes associated with Alzheimer's disease in Down syndrome. Ann Clin Trans Neurol 2018; 20: 741-51. https://doi.org/10.1002/acn3.571

[25] Rogaeva E, Meng Y, Lee J, Gu Y, Kawarai T, Zou F. The neuronal sortilin-related receptor SORT1 is genetically associated with Alzheimer's disease. Nat Genet 2007; 39: 16877.

https://doi.org/10.1038/ng1943
[26] Hochino T, Kamino K, Matsumoto M. Gene dose effect of the APOE-epsilon 4 allele on plasma HDL cholesterol level in patients with Alzheimer's disease. Neurobiol Aging 2002; 2: 41-5. https://doi.org/10.1016/S0197-4580(01)00252-4

[27] Sanchez M, Heyn S, Das D, Moghadam S, Martin K, Salehi A. Neurobiological elements of cognitive dysfunction in Down syndrome: Exploring the role of APP. Biol Psychiatry 2012; 71: 403-9. https://doi.org/10.1016/j.biopsych.2011.08.016

[28] Pritchard M, Kola I. The biological bases of pharmacological therapies in Down syndrome. In Rondal JA, Rasore Quartino A., editors. Therapies and rehabilitation in Down syndrome. Chichester, United Kingdom: Wiley 2007; pp. 18-27.

[29] Boada R, Hutaff-Lee C, Schrader A, Weitzenkamp D, Benke T, Goldson E, Costa A. Antagonism of NMDA receptors as a potential treatment for Down syndrome: A pilot randomized controlled trial. Transl Psychiatry 2012; 2e141.

\section{https://doi.org/10.1038/tp.2012.66}

[30] Matsunaga S, Kishi T, Annes P, Basun H, Hampel H, Iwata N. Lithium as a treatment for Alzheiner's disease: A systematic review and meta-analysis. J Alzheimer Dis 2015; 48: 403-10. https://doi.org/10.3233/JAD-150437

[31] Rasore Quartino, A. Le terapie attuali per le persone con sindrome di Down: La stato del arte. In Rasore Quartino A, Baccichetti $C$, editors. II presente incontra il futuro. La sindrome di Down oggi e domani. Pieve di Cadore, Italy: Tiziano Edizioni 2012; pp. 53-80

[32] Garcia-Cerro S, Rueda N, Vidal V, Lantigua S, Martinez-Cue C. Normalizing the gene dosage of DYRK1A in a mouse model of Down syndrome rescues several Alzheimer's disease phenotypes. Neurobiol Dis 2017; 106: 76-88. https://doi.org/10.1016/j.nbd.2017.06.010

[33] Kawakubo T, Mori R, Shirotani N, Iwata N, Asai M. Neprilysin is suppressed by dual-specificity tyrosine-phosphorylation regulated kinase 1A (DYRK1A) in Down syndrome derived fibroblasts. Biol Pharm Bull 2017; 40: 327-33. https://doi.org/10.1248/bpb.b16-00825

[34] Lee N, Chien Y, Hwu W. A review of biomarkers for Alzheimer's disease in Down syndrome. Neurol Ther 2017; 6: 69-81. https://doi.org/10.1007/s40120-017-0071-y

[35] Hartley S, Handen B, Devenny D, Mihaila I, Hardison R, Lao P Klunk W, Bulova P, Johnson S, Christian B. Cognitive decline and brain amyloid-beta accumulation across 3 years in adults with Down syndrome. Neurobiol Aging 2017; 58: 68-76. https://doi.org/10.1016/j.neurobiolaging.2017.05.019

[36] Motta C, Di Lorenzo F, Ponzo V, Pellicciari M, Bonni S, Picazio S, Biagio Mercuri N, Caltagirone C, Martorana A, Koch G. Transcranial magnetic stimulation predicts cognitive decline in patients with Alzheimer's disease. J Neurol Neurosurg Psychiatry 2018; 0: 1-6; published online: DOI 10.1136/jnnp-2017-317879.

[37] Kevles D. In the name of eugenics. Cambridge, Massachusetts: Harvard University Press 1999.

\section{DOI: https://doi.org/10.6000/2292-2598.2019.07.03.3}

(C) 2019 Jean A. Rondal; Licensee Lifescience Global.

This is an open access article licensed under the terms of the Creative Commons Attribution Non-Commercial License (http://creativecommons.org/licenses/by-nc/3.0/) which permits unrestricted, non-commercial use, distribution and reproduction in any medium, provided the work is properly cited. 